\title{
Crystal Structure of Hypothetical Fructose-Specific ElIB from Escherichia coli
}

\author{
Jimin Park ${ }^{1,5}$, Mi-Sun Kim ${ }^{1,5}$, Keehyung Joo ${ }^{2,5}$, Gil-Ja Jhon ${ }^{3}$, Edward A. Berry ${ }^{4}$, Jooyoung Lee ${ }^{2,}$, and \\ Dong Hae Shin ${ }^{1, *}$
}

\begin{abstract}
We have solved the crystal structure of a predicted fructose-specific enzyme IIB ${ }^{\text {tuc }}$ from Escherichia coli (EcEIIB ${ }^{\text {tuc }}$ ) involved in the phosphoenolpyruvate-carbohydrate phosphotransferase system transferring carbohydrates across the cytoplasmic membrane. EcEllB ${ }^{\text {truc }}$ belongs to a sequence family with more than 5,000 sequence homologues with $25-99 \%$ amino-acid sequence identity. It reveals a conventional Rossmann-like $\alpha-\beta-\alpha$ sandwich fold with a unique $\beta$-sheet topology. Its $C$-terminus is longer than its closest relatives and forms an additional $\beta$-strand whereas the shorter $\boldsymbol{C}$-terminus is random coil in the relatives. Interestingly, its core structure is similar to that of enzyme $I I B^{\text {cellobiose }}$ from $E$. coli $\left(E c / l B^{\text {cel }}\right)$ transferring a phosphate moiety. In the active site of the closest EcEllB $B^{\text {tuc }}$ homologues, a unique motif CXXGXAHT comprising a P-loop like architecture including a histidine residue is found. The conserved cysteine on this loop may be deprotonated to act as a nucleophile similar to that of Eclli ${ }^{\text {cel }}$. The conserved histidine residue is presumed to bind the negatively charged phosphate. Therefore, we propose that the catalytic mechanism of EcEIIB ${ }^{\text {truc }}$ is similar to that of $E c / l B^{c e l}$ transferring phosphoryl moiety to a specific carbohydrate.
\end{abstract}

\section{INTRODUCTION}

In bacteria, the phosphoenolpyruvate-carbohydrate phosphortransferase system (PTS) is a chief carbohydrate transport system (Deutscher et al., 2014) catalyzing the translocation of various carbohydrate molecules across the cytoplasmic mem-

\footnotetext{
${ }^{1}$ College of Pharmacy and Graduate School of Pharmaceutical Sciences ${ }^{3}$ Department of Chemistry and Nano Science, Global Top5 Research Program, Ewha Womans University, Seoul 03760, Korea, ${ }^{2}$ Center for insilico Protein Science and School of Computational Sciences, Korea Institute for Advanced Study, Seoul 02455, Korea, ${ }^{4}$ Department of Biochemistry and Molecular Biology, State University of New York Upstate Medical University, Syracuse, New York, USA, ${ }^{5}$ These authors contributed equally to this work.

*Correspondence: jlee@ kias.re.kr (JL); dhshin55@ewha.ac.kr (DHS)

Received 29 February, 2016; revised 21 April, 2016; accepted 27 April, 2016; published online 24 May, 2016
}

Keywords: frwD, fructose specific enzyme ElIB, functional cysteine, PTS permease, PTS system, X-ray crystallography brane. A large number of carbohydrates are phosphorylated by the PTS generally consisting of cytoplasmic proteins, enzyme $\mathrm{EI}(\mathrm{El})$ and heat stable phosphoryl carrier protein (HPr), and a membrane-associated carbohydrate-specific permease complex enzyme II (EII). In general, Ell complexes are composed of hydrophilic enzymes, EIIA and EIIB, and hydrophobic integral membrane proteins, EIIC (and EIID in a mannose- and fructose-specific PTS). The catalytic function of EI, HPr and EIIA depends on a functional histidine residue transiently phosphorylated during enzyme reaction. The active sites of EIIB subunits include either a cysteine or a histidine as a catalytic residue. The phosphoryl group which will be transferred to various carbohydrates is obtained from phosphoenolpyruvate by $\mathrm{EI}$ $\mathrm{HPr}$ becomes phosphor-HPr by accepting the phosphoryl group from El. At the next step, the phosphoryl group from phospho-HPr is transferred to a sugar-specific Ell complex. In general, El and HPr are not selective and are shared by different PTS systems. However, there are many different types of Ells present for uptake of different carbon sources, and they are not interchangeable. A phylogenetic analysis of the Ell proteins show that the PTS permease families are classified into seven families as follows (Marchler-Bauer et al., 2015); the (i) glucose (including glucoside) (Glc), (ii) fructose (including mannitol) (Fru), (iii) lactose (including $N, N$-diacetylchitobiose) (Lac), (iv) galactitol (Gat), (v) glucitol (Gut), (vi) mannose (Man), and (vii) L-ascorbate (Asc) families. In addition, PTS components directly interact with their target proteins which carry out various cellular functions such as transport proteins or transcription regulators.

An open reading frame of $E$. coli str. K-12 substr. MG1655 codes for a frwD gene (NCBI RefSeq: NP_418388.1) which is annotated as putative PTS EIIB ${ }^{\text {fruc }}$ protein $\left(E_{C} E\right.$ II $\left.^{\text {fruc }}\right)$ of 12.6 $\mathrm{kDa}$. It belongs to the $\mathrm{NCBI}$ conserved domain cd05569 (PTS_IIB_fructose) which includes subunit IIB of Ell of the fructose specific PTS (Barabote and Saier, 2005). A PSI-BLAST search (http://www.ncbi.nlm.nih.gov) of this sequence revealed around six hundred proteins with sequence identity above $35 \%$ (Fig. 1). Most of the homologous sequences are annotated as fructose-like specific PTS system EIIB or fused forms, ElIBC or EIIABC. The biochemical study revealed that at least 15 different Ell complexes are found in Escherichia coli (Saier and Reizer, 1994). Several putative fructose-like IIB components are also detected in the E. coli PTS system. Since the molecular structure and the functional implication of this EcElIB fruc family have not been reported, we have determined the X-ray crystal structure of $E c E^{\prime I I}{ }^{\text {fruc }}$. Based on the crystal structure analy- 


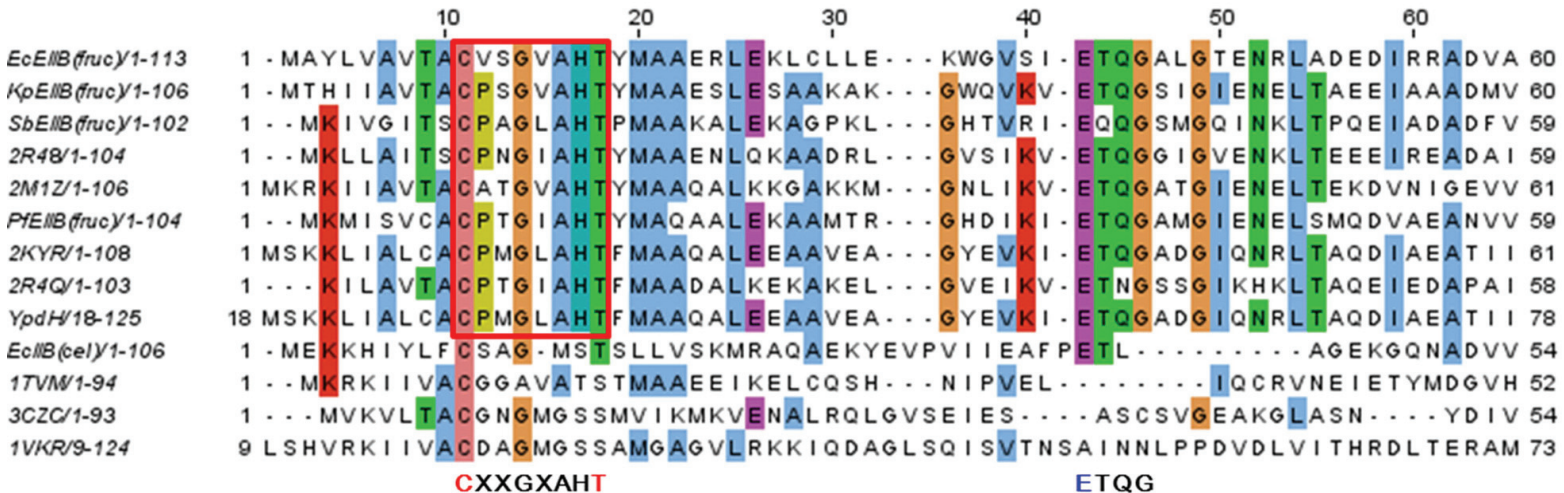

EcEIIB(fruc)
GOT Model
EcIIB(cel)

P1

EcEMB fruc) y1-113 KoEMB fruc) 1-106 SbEMB firuc Y 1-102 2R48/104 2M1Z1-106 PIEMB (fruc) 1-104 2KYR/1-108 2R4Q1-103 YodH/18-125 EcMB(cel)1-106 1TVNS1-94 3CZC/1-93 1VKR/9-124

EcEIIB(fruc)

GOT Model

EcllB(cel)
70
100

110

120

61 LLITDIELAGAERFE - . HCRYVQCSIYAFLR - EPQRVMSAVRKVLSAPQQTHLILE $100 \%$ 61 ILTKDIGIKFEERFA. . GKT IVRVNISDAVK.RADAIMNKI DSHLSQSA..... 60 LLATDQKIVGEERFE. . GKKKIRVNISTCIK. APEAVLKKCIQAVS . . . . . . . . . . . 60 I I AADRSVN - KDRF I . . GKKLLSVGVQDG IR - KPEELIQKALNGDIPVY..... $34 \%$ 62 I FAVDTKVRNKERFD... GKVVLEVPVSAPIK. DAEKVINAALAL I DEK...... $34 \%$ 60 I F AVGVNVEGEERFH . . GKPS IMVEVGKAIE - NPVAVIEKAEK. KGEEV..... $34 \%$ 62 I HSVAVTPEDNERFE . . SRDVYEITLQDAIK-NAAG I I KEIEEMIASEQQ . . . . $34 \%$ 59 I VAADKQVE - MERFK. - GKRVLQVPVTAG IR - RPQEL IEKAMNQDAPIY. . . . . $34 \%$ 79 I HSVAVTPEDNERFE. . SRDVYEITLQDAIK. NAAG I I KEIEEMIASEQQ . . . . 32\% 55 LLGPQ I AYMLPEIQRLLPNKPVEVIDSLLYGK. VDGLGVLKAAVAAIKKAAAN - . $21 \%$ 53 L I CTTARVD. . RSFG. . DIPLVHGMPFVSGVG. IEALQNKILTILQG...... 55 VASNHL I HELDGRTN - . GKL IGL - DNLMDDNE - IKTKLEEA . . . KK. . . . . 74 RQVPQAQH I SLTNFLDSGLYTSLTE - RLVAAQRHTANEEKVKDSLKDSFDDS . . . $7 \%$ ERF

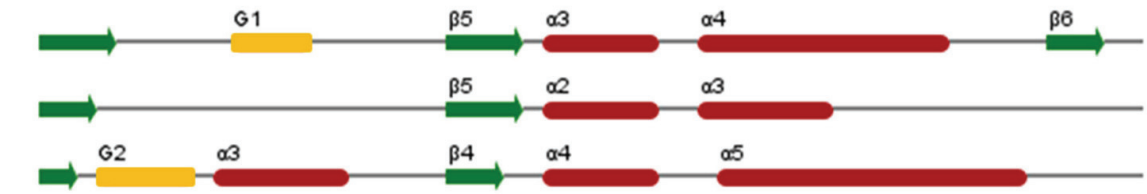

Fig. 1. Multiple sequence alignment of $E c E E_{I I}^{\text {fruc }}$ and its homologues. Abbreviations are as follows: Predicted EllB ${ }^{\text {fruc }}$ molecules from Escherichia coli str. K-12 substr. MG1655 (EcEIIB(fruc)), Klebsiella pneumoniae 342 (KpEIIB(fruc)), Streptococcus bovis ATCC 700338 (SbEIIB(fruc)), Bacillus Subtilis Subsp. Subtilis Str. 168 (2R48), Listeria monocytogenes EGD-e (2M1Z), Pelosinus fermentans DSM 17108 (PfEIIB(fruc)), E. coli K-12 (2KYR), Bacillus subtilis subsp. subtilis str. 168 (2R4Q), E. coli UTI89 (YpdH), EcllB ${ }^{\text {cel }}$ (EcllB(cel)), GatB E. coli from (1TVM), PtxB from Streptococcus (3C2Z) and the cytoplasmic B domain of the mannitol transporter IIABC from E. coli (1VKR). Secondary structure elements of $E c E I_{I B} B^{\text {fruc }}$, the GOT model and $E C$ IIB $^{\text {cel }}$ are drawn below the sequences and colored red for $\alpha$-helix, green for $\beta$-strand and yellow for $3_{10}$-helix. The dotted lines represent the residues missing in the coordinates. The highly conserved motifs are indicated. The conserved sequences at the P-like loop motif are marked with a red box. The red characters represent the key residues for transferring a phosphoryl group. Glu38 and Arg73 forming a salt bridge are marked as the blue characters. The consensus amino acids are colored according to the Clustal $X$ color scheme provided by the Jalview program (Waterhouse et al., 2009).

sis, molecular functions of EcEllB ${ }^{\text {fruc }}$ have been inferred and discussed in this paper.

\section{MATERIALS AND METHODS}

\section{Structure determination}

Details of the preliminary X-ray study (Shin, 2008) and the structure determination (Joo et al., 2015) of EcEllB fruc had been previously published. In brief, EcEllB fruc crystals were grown using 20\% (w/v) PEG MME2000 in the presence of $10 \mathrm{mM}$ nickel (II) chloride hexahydrate. The X-ray dataset of EcEIIB ${ }^{\text {fruc }}$ was collected to $2.28 \AA$ resolution and the crystal belonged to the primitive trigonal space group $P 3_{2}$ with unit-cell parameters $a=b=33.11, c=154.38 \AA$. Molecular replacement (MR) was performed using PHENIX (Adams et al., 2010) with highly accurate protein $3 \mathrm{D}$ models generated by the recently proposed method called GOT (Global-Optimization-based Templatebased modeling of proteins) (Joo et al., 2015). A relatively good electron density map was obtained after automated modelbuilding and refinement with the PHENIX AutoBuild wizard 
A

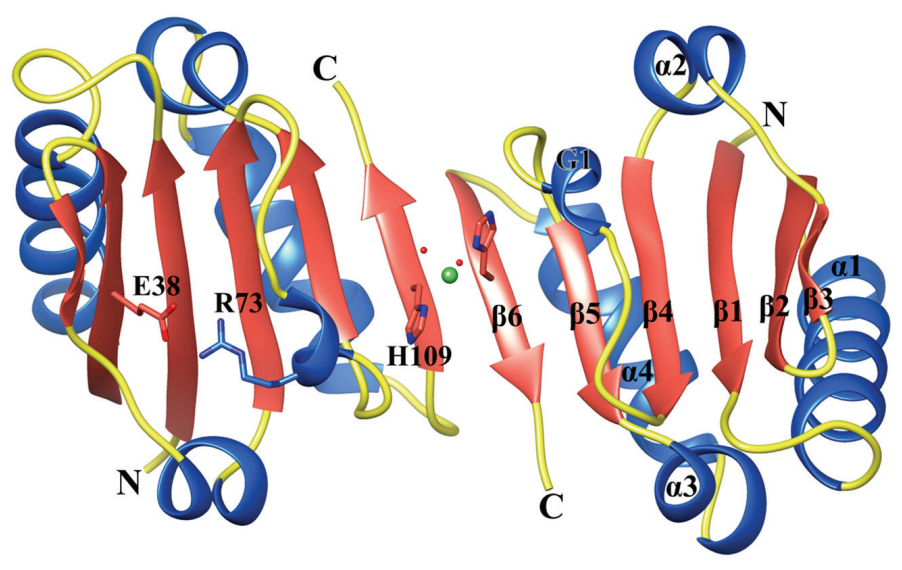

B

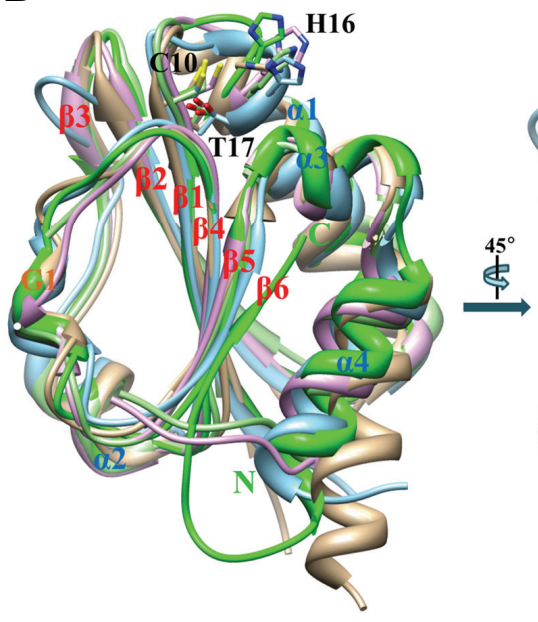

Fig. 2. (A) The crystal structure of dimeric $E C E E^{\text {firuc }}$. The secondary structural elements of $E c E l l B^{\text {fruc }}$ are labeled. The two water molecules (red) and two histidine residues coordinating to nickel ion (green) are drawn. Glu38 and Arg73 forming a salt bridge with a distance of $\sim 2.7 \AA$ are also drawn. (B) The superimposed crystal structures of EcEllB fruc (green) and its closest homologues, 2R48 (Plum), 2R4Q (Light green), 2M1Z (Sky blue) and 2KYR (Tan), in two different views related by a $45^{\circ}$ rotation. The secondary structural elements of $E c E{ }^{\prime} B^{\text {fruc }}$ are labeled. Some $\mathrm{C}$-terminals are labeled according to their ribbon colors. The potential active site residues, cysteine, histidine and threonine residues are drawn with a stick model.
(Afonine et al., 2012). The electron density map clearly showed the presence of two protomers of $E_{C} E_{I I B}{ }^{\text {fruc }}$ in the asymmetric unit. Interestingly, they form a dimer bridged by a nickel ion contained in the crystallization solution. The final model exhibited good stereochemical geometry and was refined to R- and Rfree values of $22.5 \%$ and $29.9 \%$, respectively (Joo et al., 2015). The atomic coordinates and structure factors of the $E_{C} E_{I I B}{ }^{\text {fruc }}$ structure have been deposited in the RCSB Protein Data Bank under the accession code 4TN5.

\section{RESULTS AND DISCUSSION}

The crystal structure of EcEIII ${ }^{\text {fruc }}$ has been determined at the resolution of $2.4 \AA$ (Supplementary Fig. S1). The EcElIB ${ }^{\text {fruc }}$ structure includes all residues of the original sequence. The structure of the $E_{C} E_{I I B^{\text {fruc }}}$ protein is composed of a central sixstranded parallel open twisted $\beta$-sheet, which is flanked by three $\alpha$-helices $(\alpha 1, \alpha 3, \alpha 4)$ on the concave side and two $(\alpha 2$, G1) on the convex side (Fig. 2A). In the asymmetric unit, the crystal structure shows an artificial dimer seemingly being triggered by one nickel ion coordinated to two neighboring His109 residues located on the $\mathrm{C}$-terminus of each protomer (Fig. 2A). This coordination may induce a loop-to-strand conversion of the C-terminal loop resulting in the formation of the last $\beta$-strand $\beta 6$. As a result, a twelve stranded $\beta$-sheet composed of two contig- uous $\beta$-sheets is formed in the dimer. However, $E c E$ III $^{\text {fruc }}$ is a monomer as indicated in the monodisperse peak with molecular weight of $13 \mathrm{kDa}$ from Dynamic light scattering (DynaPro 99, Proterion Corporation, USA) (Shin, 2008) and this dimeric form is not found in the other crystal structures of its closest homologues as mentioned below. It is noteworthy that the addition of zinc ions produced same kinds of crystals. However nickel ions improved the diffraction limits of crystals (Shin, 2008). In the crystal structure, two histidine residues and two water molecules coordinated the nickel ion. During the refolding step, various metals were tried but a dimeric size was not detected in size exclusion chromatography and dynamic light scattering. Furthermore, the CD spectrum showed the same secondary structure contents regardless of the presence of metal ions including zinc and nickel ions (Oganesyan et al., 2005). However, the metal induced dimerization of EcEllB ${ }^{\text {fruc }}$ cannot be thoroughly excluded and its biorelevance should be further studied inside bacterial cells.

$E_{C} E$ IB $^{\text {fruc }}$ belongs to a sequence family with more than 5000 sequence homologues having $25-99 \%$ amino-acid sequence identity obtained by a PSI-BLAST search (Fig. 1). The 3D structure alignment search with Dali (Holm and Rosenstrom, 2010) reveals that the crystal structure of $E_{C} E_{I I I}{ }^{\text {fruc }}$ determined in this study, along with the closest homologues (Z-scores above 14), 2R48 (mannose-specific ElIB from Bacillus subtilis), 2R4Q 
A

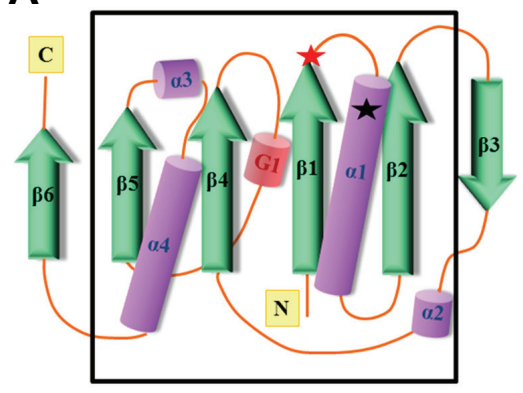

B

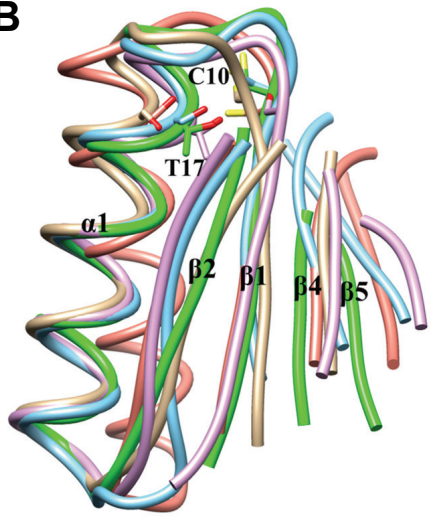

Fig. 3. (A) Topology diagram is shown. $\alpha$ helices are represented by purple cylinders, $\beta$ strands by green arrows, and $3_{10}$-helix by a red cylinder. Secondary structure elements of helices and strands are labeled. Cys 10 and Thr17 are represented by red and black stars, respectively. The boxed region represents the topology overlapped with $\mathrm{EClIB}^{\mathrm{cel}}$. (B) The superimposed core structures of $E c E$ IIB $^{\text {fruc }}$ (green) and $\mathrm{CClIB}^{\text {cel }}$ homologues, 1IIB (Plum), 1TVM (Tan), 1VKR (Sky blue) and 3CZC (Salmon). The secondary structural elements of $E c E$ IIB $^{\text {fruc }}$ are labeled. The potential active site residues, cysteine and threonine residues are drawn with a stick model. (C) The superimposed core structures of EcElli fruc (green) and the low-molecular-weight PTPase (plum) from Bos taurus (PDB ID: 1PHR). The potential active site residues of both proteins are labeled and drawn with a stick model. Their catalytic loops are depicted in the solid box. The zoomed view represents the detailed view of the P-like loop of EcEllB fruc and the P-loop of lowmolecular-weight PTPase. The residues of consensus sequence are drawn with a stick model. (fructose-specific EIIB from B. subtilis), 2M1Z (hypothetical protein Imo0427 from Listeria monocytogenes) and 2KYR (fructose-like enzyme IIB from $E$. coli), forms a unique fold family not found in other protein structures. It is composed of $\beta 1, \alpha 1, \beta 2$, $\beta 3, \alpha 2, \beta 4, \mathrm{G} 1, \beta 5, \alpha 3$ and $\alpha 4$. The salt bridge formed by Glu38 and Arg73 (Fig. 2A) from two highly conserved sequence motifs, ${ }^{38} \mathrm{ETQG}^{41}$ and ${ }^{72} \mathrm{ERF}^{74}$ (Fig. 1), seems to stabilize the overall architecture of the family. The root-mean-square (rms) deviations of matching $\mathrm{C} \alpha$ atom positions of $E c E$ III $^{\text {fruc }}$ compared with its closest homologues are $0.727 \AA^{2}$ (70 C $\alpha$ atoms of 2R48) $0.965 \AA^{2}$ (71 of $2 \mathrm{R} 4 \mathrm{Q}$ ), $1.090 \AA^{2}$ (86 of $2 \mathrm{M} 1 \mathrm{Z}$ ) and 0.989 $\AA^{2}(80$ of $2 K Y R)$, respectively. It is noteworthy that the Cterminus of $E C E E^{\prime} B^{\text {fruc }}$ is longer than its closest homologues and forms an additional $\beta$-strand $\beta 6$ whereas the shorter $C$-termini of the other members are unstructured (Fig. 2B). As a result, the central $\beta$-sheet of $E c E_{I I B}{ }^{\text {fruc }}$ has the topology of $6 \uparrow 5 \uparrow 4 \uparrow 1 \uparrow$ $2 \uparrow 3 \downarrow$ instead of $5 \uparrow 4 \uparrow 1 \uparrow 2 \uparrow 3 \downarrow$ found in the other members. The Dali search revealed that the topology of the six-stranded $\beta$-sheet, $6 \uparrow 5 \uparrow 4 \uparrow 1 \uparrow 2 \uparrow 3 \downarrow$, is unique and not detected in other protein structures. Although this additional $\beta$-stand is stabilized in our crystal form by an inter-molecular antiparallel $\beta$-bonding and chelation of a nickel ion by the neighboring two His108 (Fig. $2 \mathrm{~A}$ ), it is not yet clear whether the last $\beta$-strand is an intrinsic structure or an artificial result. In fact, the predicted secondary structure of the C-terminus of EcElli ${ }^{\text {fruc }}$ calculated with PSIPRED (http://bioinf.cs.ucl.ac.uk/psipred/) (Buchan et al.,
2013) is a loop. Therefore, the state of the C-terminus in solution and the possibility of a transition from disordered to ordered or vice versa related to its function are still under investigation. The next homologue group of $E_{C} E$ II $^{\text {fruc }}$ is DNA-binding response regulators with Z-scores above 8 . However, this family has a different $\beta$-sheet topology from EcEIIB ${ }^{\text {fruc }}$ and contains unique active site residues. The structure of the ElIB subunit of fructose permease (ElIB $\left.{ }^{\text {Lev }}\right)$, another homologue of $E c E E_{I I} B^{\text {fruc }}$, also has a different $\beta$-sheet topology $(3 \uparrow 2 \uparrow 4 \uparrow 1 \uparrow 5 \uparrow 6 \uparrow 7 \downarrow)$ with the active site residue, His15 (Schauder et al., 1998).

Interestingly, the Dali search revealed that the core $\beta$-sheet topology, $2 \uparrow 1 \uparrow 4 \uparrow 5 \uparrow$, of $E C E$ III $^{\text {fruc }}$ is closer to that of enzyme IIB $^{\text {cellobiose }}$ from $E$. coli $\left(E C_{\text {IIB }}{ }^{\text {cel }}\right.$, PDB: 1 III) (van Montfort et al., 1997) with the Z-score of 7.2 (Fig. 3A). It contains a $\beta$-sheet with the topology of $2 \uparrow 1 \uparrow 3 \uparrow 4 \uparrow$ lacking two $\beta$-strands at each end of the sheet. EClIB ${ }^{\text {cel }}$ homologues comprises a protein family with the enzyme GatB of the galactitol-specific phosphoenolpyruvate-dependent phosphotransferase system from $E$. coli (1TVM), the cytoplasmic B domain of the mannitol transporter IIABC from $E$. coli (1VKR), and PtxB from Streptococcus (3CZC). They have the $\beta$-sheet topology of $2 \uparrow 1 \uparrow 3 \uparrow 4 \uparrow$ with a conserved cysteine (Cys10) and a threonine/serine (Thr17) located at the active site constructed by a P-like loop (Fig. 3B) as mentioned below (Su et al., 1994). The cysteine residue functions via a cysteine-phosphate intermediate and the threonine residue assists the binding of phosphate (Ab et al., 1997). 
The multiple sequence alignment shown in Fig. 1 indicates that these two residues are also conserved among EcElIB ${ }^{\text {fruc }}$ homologues. Since these residues are critical in transferring a phosphoryl group in EClIB ${ }^{\text {cel }}$ homologues, Cys10 and Thr17 of ECEIIB ${ }^{\text {fruc }}$ may function in a similar way.

As for $E_{C I I B}{ }^{\text {cel }}$ homologues, the phosphorylation site of EIIB is located on this conserved cysteine residue at the $\mathrm{N}$-terminal end of the P-like loop. This cysteine receives the phosphoryl group from EIIA and transfers it to the specific carbohydrate when bound at the catalytic site of EIIC. The catalytic activity of the cysteine is aided by the P-like loop structurally similar to that of the phosphate binding P-loop of the phosphotyrosine protein phosphatase (PTPase) superfamily (consensus sequence CXXXXXR(S/T)) (Su et al., 1994). The P-loop forms an anionbinding motif together with the helix dipole of helix $\alpha 1$ and thus provides a favorable environment to generate deprotonated cysteine as a nucleophile and to accommodate a negatively charged phosphoryl group. The conserved arginine residue in the consensus sequence supports the catalytic process. However, the $\mathrm{P}$-like loop of $\mathrm{EClB}^{\mathrm{cel}}$ homologues lack the conserved arginine residue at the corresponding position of the P-loop of the PTPase superfamily and thus the insufficient positive charge is thought to be compensated by their partner molecules (Lei et al., 2009).

As mentioned above, the architecture of the core structure of $E c E I I B^{\text {fruc }}$ is quite similar to the overall structure of $E c I^{\text {cel }}$ together with the conserved residues, Cys10 and Thr17. When the core structural elements of $E_{C} E I_{I B}$ fruc are superimposed with those of EClIB ${ }^{\text {cel }}$ homologues (Fig. 3B), the rms deviations of aligned $\mathrm{C} \alpha$ atoms are $0.822 \AA^{2}\left(25 \mathrm{C} \alpha\right.$ atoms of 1 IIIB) $0.929 \AA^{2}$ (28 of 1TVM), $1.030 \AA^{2}$ (32 of 1VKR) and $1.253 \AA^{2}$ (10 of $3 \mathrm{CZC}$ ), respectively. However, unlike EcllB ${ }^{\text {cel }}$, EcElli ${ }^{\text {fruc }}$ homologues contain a conserved histidine His16 which may assist accommodating a negatively charged phosphoryl group. The spatial position occupied by His 16 in the P-like loop is almost equivalent to that of the functional arginine of the P-loop of the PTPase superfamily (Fig. $3 \mathrm{C}$ ). Consequently, the EcElIB ${ }^{\text {fruc }}$ family has a unique consensus sequence of $C X X G X A H T$ at the P-like loop. Interestingly, the crystal structures of the nucleotide binding subunit $B$ of $A_{1} A_{0}$ ATP synthase (Sankhala et al., 2014) and human PIR1 (Tadwal et al., 2012) revealed that they also contain a catalytic histidine residue in their P-loops which interacts with a phosphate moiety.

In summary, we have determined the crystal structure of $E c E_{I I B}{ }^{\text {fruc }}$. The Dali search revealed that its core structure resembles that of EClIB ${ }^{\text {cel }}$. Though the overall fold is quite similar to different IIB enzymes and other fold families, EcEIIB ${ }^{\text {fruc }}$ homologues still consist of an independent subfamily due to their unique topological connection. Based on the sequence alignment and structural comparison of its homologues, a unique motif CXXGXAHT comprising a P-loop like architecture is also inferred. Therefore, we proposed that the conserved cysteine on this loop may be deprotonated to act as a nucleophile to transfer phosphoryl moiety to a specific carbohydrate. The bigger size of EcEIIB ${ }^{\text {fruc }}$ homologues together with the presence of extra catalytic histidine over EcllB ${ }^{\text {cel }}$ suggests that though their core molecular function is similar, their biological partners and substrates may be different. A further study is going on to find a biological partner and substrate of $E_{C} E_{I I} B^{\text {fruc }}$.

Note: Supplementary information is available on the Molecules and Cells website (www.molcells.org).

\section{ACKNOWLEDGMENTS}

We are grateful to the staffs at Pohang Light Source and Ad- vanced Light Source. We also thank KIAS Center for Advanced Computation for providing Linux cluster system for computational modeling. This work was supported by the Core Research Center program (2012-0000952), the Converging Research Center Program (2010K000266) and the Basic Science Research Program (2008-331-C00233, 2010-0003907, 2013R 1A1A1A05008769) through the National Research Foundation of Korea grant funded by the Ministry of Education, Science and Technology, Republic of Korea (MEST). M.-S. Kim and J. Park were supported by the Brain Korea 21 (BK21) Project.

\section{REFERENCES}

Ab, E., Schuurman-Wolters, G., Reizer, J., Saier, M.H., Dijkstra, K., Scheek, R.M., and Robillard G.T. (1997) The NMR side-chain assignments and solution structure of enzyme IIB of the phosphoenolpyruvate-dependent phosphotransferase system of Escherichia coli. Protein Sci. 6, 304-314.

Adams, P.D., Afonine, P.V., Bunkoczi, G., Chen, V.B., Davis, I.W., Echols, N., Headd, J.J., Hung, L.W., Kapral, G.J., GrosseKunstleve, R.W., et al. (2010). PHENIX: a comprehensive Python-based system for macromolecular structure solution. Acta Crystallogr. Sect D: Biol. Crystallogr. 66, 213-221.

Afonine, P.V., Grosse-Kunstleve, R.W., Echols, N., Headd, J.J., Moriarty, N.W., Mustyakimov, M., Terwilliger, T.C., Urzhumtsev, A., Zwart, P.H., and Adams, P.D. (2012). Towards automated crystallographic structure refinement with phenix.refine. Acta Crystallogr. Sect D: Biol. Crystallogr. 68, 352-367.

Barabote, R.D., and Saier, M.H., Jr. (2005). Comparative genomic analyses of the bacterial phosphotransferase system. Microbiol. Mol. Biol. Rev. 69, 608-634.

Buchan, D.W., Minneci, F., Nugent, T.C., Bryson, K., and Jones, D.T. (2013). Scalable web services for the PSIPRED Protein Analysis Workbench. Nucleic Acids Res. 41, W349-357.

Deutscher, J., Ake, F.M., Derkaoui, M., Zebre, A.C., Cao, T.N., Bouraoui, H., Kentache, T., Mokhtari, A., Milohanic, E., and Joyet, P. (2014). The bacterial phosphoenolpyruvate:carbohydrate phosphotransferase system: regulation by protein phosphorylation and phosphorylation-dependent protein-protein interactions. Microbiol. Mol. Biol. Rev. 78, 231-256.

Holm, L., and Rosenstrom, P. (2010). Dali server: conservation mapping in 3D. Nucleic Acids Res. 38, W545-549.

Joo, K., Kim, M.-S., Park, J., Lee, J., and Shin, D.H. (2015). Highaccuracy protein structure modeling and its application to molecular replacement of crystallographic phasing. Biodesign. 3, 123-130.

Lei, J., Li, L.F., and Su, X.D. (2009). Crystal structures of phosphotransferase system enzymes PtxB (IIB(Asc)) and PtxA (IIA(Asc)) from Streptococcus mutans. J. Mol. Biol. 386, 465-475.

Marchler-Bauer, A., Derbyshire, M.K., Gonzales, N.R., Lu, S., Chitsaz, F., Geer, L.Y., Geer, R.C., He, J., Gwadz, M., Hurwitz, D.I., et al. (2015). CDD: NCBl's conserved domain database. Nucleic acids Res. 43, D222-226.

Oganesyan, N., Kim, S.-H., and Kim, R. (2005). On-column protein refolding for crystallization. J. Struct. Funct. Genomics 6, 177-182

Saier, M.H., Jr., and Reizer, J. (1994). The bacterial phosphotransferase system: new frontiers 30 years later. Mol. Microbiol. 13, 755-764.

Sankhala, R.S., Lokareddy, R.K., and Cingolani, G. (2014). Structure of human PIR1, an atypical dual-specificity phosphatase. Biochemistry 53, 862-871.

Schauder, S., Nunn, R.S., Lanz, R., Erni, B., and Schirmer, T. (1998). Crystal structure of the IIB subunit of a fructose permease (IIBLev) from Bacillus subtilis. J. Mol. Biol. 276, 591-602.

Shin, D.H. (2008). A preliminary X-ray study of a refolded PTS EllBfruc protein from Escherichia coli. Protein Pept. Lett. 15, 630632.

Su, X.D., Taddei, N., Stefani, M., Ramponi, G., and Nordlund, P. (1994). The crystal structure of a low-molecular-weight phosphotyrosine protein phosphatase. Nature 370, 575-578.

Tadwal, V.S., Sundararaman, L., Manimekalai, M.S., Hunke, C., and Gruber, G. (2012). Relevance of the conserved histidine and asparagine residues in the phosphate-binding loop of the nucleo- 
tide binding subunit $B$ of $A(1) A(0)$ ATP synthases. J. Struct. Biol. 180, 509-518.

van Montfort, R.L., Pijning, T., Kalk, K.H., Reizer, J., Saier, M.H., Jr., Thunnissen, M.M., Robillard, G.T., and Dijkstra, B.W. (1997). The structure of an energy-coupling protein from bacteria, IIBcellobiose, reveals similarity to eukaryotic protein tyrosine phospha- tases. Structure 5, 217-225.

Waterhouse, A.M., Procter, J. B., Martin, D.M., Clamp, M., and Barton, G.J. (2009). Jalview Version 2--a multiple sequence alignment editor and analysis workbench. Bioinformatics 25, 1189-1191. 